\title{
Hepatic and HSC-specific sorafenib effects in rats with established secondary biliary cirrhosis
}

\author{
Martin Hennenberg ${ }^{1}$, Jonel Trebicka ${ }^{1}$, Zaki Kohistani ${ }^{1}$, Christian Stark ${ }^{1}$, Hans-Dieter Nischalke ${ }^{1}$, \\ Benjamin Krämer ${ }^{1}$, Christian Körner ${ }^{1}$, Sabine Klein ${ }^{1}$, Michaela Granzow ${ }^{1}$, Hans-Peter Fischer ${ }^{2}$, \\ Jörg Heller ${ }^{1}$ and Tilman Sauerbruch ${ }^{1}$
}

Portal hypertension in cirrhosis depends on increased intrahepatic vascular resistance, which is explained by fibrosis and intrahepatic hyperresponsiveness to vasoconstrictors. Both are caused by activation and proliferation of hepatic stellate cells (HSCs). Portal hypertension of cirrhotic rats can be reduced by the multikinase inhibitor sorafenib, due to a reduction of intrahepatic vascular resistance. Therefore, the hepatic effects of sorafenib require further understanding. Here, we investigated hepatic and HSC-specific sorafenib effects in cirrhotic rats. Animal models of bile duct ligation-induced secondary biliary cirrhosis in rats were studied. The rats were treated with sorafenib $(60 \mathrm{mg} / \mathrm{kg} / \mathrm{day})$ for 1 week, starting after established cirrhosis. Histological evaluation was carried out using hemalaun and eosin (HE) staining. Apoptosis was studied by PARP cleavage, colorimetric caspase-3 assay, and electrophoretic DNA detection. HSC activation was studied by hepatic Sirius red and immunohistochemical $\alpha$ SMA ( $\alpha$-smooth muscle actin) staining, and by in vitro experiments with culture-activated primary HSCs. Biochemical serum parameters suggested the occurrence of sorafenib-induced liver damage. $\mathrm{HE}$ staining revealed histological changes in livers of sham-operated and bile duct-ligated (BDL) rats in response to sorafenib, which were different in both groups. In BDL rats and isolated HSCs, the treatment with sorafenib reduced hepatic $\alpha \mathrm{SMA}$ and procollagen- $1 \alpha$ mRNA expression. As shown by immunohistochemical staining, perisinusoidal $\alpha \mathrm{SMA}$ expression was reduced by sorafenib in BDL rats. This was associated with reduced perisinusoidal deposition of extracellular matrix, as revealed by Sirius red staining. Although no change in PARP cleavage and only a minor increase in hepatic caspase-3 activity were detected in BDL rats in response to sorafenib, livers of sorafenib-treated BDL rats contained small DNA fragments, which were not observed in untreated BDL rats. In conclusion, sorafenib treatment reduces the number of activated HSCs in cirrhotic livers. This leads to the decrease in intrahepatic vascular resistance, but also to liver damage in the dosage we used. Therefore, any translation to portal hypertensive patients who may profit from sorafenib should be done with particular care.

Laboratory Investigation (2011) 91, 241-251; doi:10.1038/labinvest.2010.148; published online 4 October 2010

KEYWORDS: cirrhosis; fibrosis; hepatic stellate cell; portal hypertension; sorafenib

Portal hypertension is responsible for severe complications of liver cirrhosis. ${ }^{1}$ The increased portal pressure is partially explained by an increased intrahepatic vascular resistance, due to intrahepatic fibrosis and hyperresponsiveness to vasoconstrictors. $^{2-6}$ For both, activation of hepatic stellate cells (HSCs) and transdifferentiation to a myofibroblastlike, highly proliferative phenotype has a key role. ${ }^{2-6}$ After their phenotypic switch, HSCs acquire contractility and become a major source of extracellular matrix (ECM) production. $^{2-6}$
The multikinase inhibitor sorafenib was tested for the treatment of hepatocellular carcinoma (HCC). ${ }^{7}$ It improves survival in patients with advanced tumors and compensated cirrhosis. ${ }^{7}$ Furthermore, it has been recently demonstrated that treatment with sorafenib reduces portal hypertension in rats with experimentally induced cirrhosis. ${ }^{8,9}$ This resulted from a diminished intrahepatic vascular resistance, assumed to be caused by an effect of sorafenib on HSCs. ${ }^{8,9}$ This suggests nontumor-related effects of sorafenib in cirrhosis. Here, we investigated liverand HSC-specific effects of sorafenib in cirrhotic rats.

\footnotetext{
${ }^{1}$ Department of Internal Medicine I, University of Bonn, Bonn, Germany and ${ }^{2}$ Department of Pathology, University of Bonn, Bonn, Germany Correspondence: Dr M Hennenberg, PhD, Urologisches Forschungslabor, LIFE-Zentrum, Marchioninistraße 23, München 81377, Germany. E-mail: martin.hennenberg@med.uni-muenchen.de 


\section{METHODS}

\section{Animals}

Secondary biliary cirrhosis in male Sprague-Dawley rats (Charles River, Sulzfeld, Germany) was induced by bile duct ligation (BDL), as previously described. ${ }^{2,3,8}$ In brief, the common bile duct of rats, each with an initial body weight of $\sim 200 \mathrm{~g}$, was exposed after median laparotomy and ligated twice. The segment between the two ligations was resected and the abdomen was sutured. Experiments were performed 4-5 weeks after BDL, when cirrhosis with ascites was present. Sham-operated rats served as controls. In these rats, the common bile duct was exposed, but no ligation or resection was performed. The study was approved by the local committee for animal studies (District Government, 9.93.2.10.31.07.140).

\section{Treatment and Sampling}

At 4-5 weeks after BDL, when cirrhosis with ascites was present, cirrhotic rats were randomly divided into two groups. Rats of one group were treated with the multikinase inhibitor sorafenib $(60 \mathrm{mg} / \mathrm{kg}$ body weight/day) for 7 days by gavage, whereas the remaining group was treated with solvent. For the treatment, we used sorafenib (Nexavar, $200 \mathrm{mg}$ ) from Bayer Healthcare (Leverkusen, Germany). After removal of the outer coat, pills were ground up in a tissue mill. The resulting powder was mixed with tap water and applied via gavage to rats. This treatment has been shown to be effective, and it reduces portal pressure. ${ }^{8}$ For the treatment with solvent, rats received tap water instead of sorafenib suspension. The applied volume of sorafenib suspension or solvent was $1 \mathrm{ml}$ for each dose.

For sampling, excised livers were transferred to oxygenized Krebs-Henseleit solution, in which they were cut into samples. These were either immediately shock frozen in liquid nitrogen and subsequently kept for further analyses or directly transferred into buffered 10\% formaldehyde solution for paraffin embedding.

\section{Analysis of Biochemical Serum Parameters}

Blood was withdrawn from the inferior vena cava and analysis of biochemical serum parameters was performed using standard methods.

\section{RT-PCR}

RNA isolation, reverse transcription, and RT-PCR were performed as previously described., ${ }^{2,3}$ In brief, RNA was isolated from $30 \mathrm{mg}$ shock-frozen liver tissue with the RNeasy-mini kit (Qiagen, Hilden, Germany) according to the manufacturer's guidelines. RNA concentrations were measured spectrophotometrically at $260 \mathrm{~nm}$. For each sample, $1 \mu \mathrm{g}$ total RNA was used. Before reverse transcription, samples were DNA-digested by incubation with RQ1 RNase-free DNAse (Promega, Madison, USA). Reverse transcription was performed using MMLV reverse transcriptase (Invitrogen, Karlsruhe, Germany) and random primers (250 ng,
Microsynth, Balgach, Switzerland). Control reactions did not contain reverse transcriptase. Primers and probes for detection of targets and house-keeping gene (18SrRNA) were provided by Applied Biosystems (Foster City, USA) as readyto-use mixes and used according to the manufacturer's guidelines. RT-PCR was performed using the ABI 7700 sequence detector (Applied Biosystems). The PCR reaction was performed in a volume of $25 \mu \mathrm{l}$ containing $12.5 \mu \mathrm{l}$ of $2 \times$ TaqMan PCR master mix (Roche Molecular Systems, Mannheim, Germany/Applied Biosystems) and $2 \mu \mathrm{l}$ (equivalent to $67 \mathrm{ng}$ total RNA) of cDNA. 18SrRNA served as endogenous control; the final concentrations were $100 \mathrm{nM}$ for primers and $200 \mathrm{nM}$ for the probe. The results were expressed as the number of cycles (Ct value) at which the fluorescence signal exceeded a defined threshold. The difference in $\mathrm{Ct}$ value of the target cDNA and the endogenous control are expressed as negative $\Delta C \mathrm{t}$ values. Therefore, higher $-\Delta C \mathrm{t}$ values denote higher mRNA levels. Data are presented as $-\Delta C \mathrm{t}$, or $2^{-\Delta \Delta C \mathrm{t}}$. For all the target genes and for 18SrRNA, a validation experiment was performed according to the manufacturer's guidelines. In these experiments, it was demonstrated that the efficiencies of the RT-PCR for the target gene and the endogenous control were approximately equal. Therefore, the $\Delta C t$ method could be used for relative quantification.

\section{Western Blot Analysis}

Western blot analysis was performed as previously described. $^{2,3,8}$ In detail, the following primary antibodies were used: anti- $\alpha$-smooth muscle actin (anti- $\alpha$ SMA; Sigma Aldrich, Schnelldorf, Germany); anti-PARP (BD Transduction Laboratories, Heidelberg, Germany). After incubation with appropriate peroxidase-coupled secondary antibodies, detection was performed with enhanced chemiluminescence (Amersham, Amersham, UK). Films were developed using a Kodak X-omat (Kodak, Stuttgart, Germany). Densitometric quantification was performed using a FLA-300 phosphoimager (Fuji Film, Dusseldorf, Germany).

\section{Histological Staining}

Hemalaun and eosin staining (HE staining) for histological evaluation of paraffin-embedded liver sections $(3-4 \mu \mathrm{m})$ was performed using standard methods. For quantification of cells in stained sections, nuclei in parenchymal regions were counted in five different high-power fields of each sample. For the detection of collagen fibers, paraffin-embedded liver sections were stained with Sirius red using standard methods. Thus, sections $(3-4 \mu \mathrm{m})$ were stained in $0.1 \%$ Sirius Red F3B in saturated picric acid (Chroma, Münster, Germany).

\section{Immunohistochemistry}

Sections from paraffin-embedded tissues $(3-4 \mu \mathrm{m})$ were stained by an indirect immunoperoxidase technique, as described previously. ${ }^{2}$ In brief, endogenous peroxidase activity was blocked by $0.03 \% \mathrm{H}_{2} \mathrm{O}_{2} / \mathrm{NaNO}_{3}$. The sections were incubated with primary mouse monoclonal antibody 
against $\alpha$ SMA (1:600) (Sigma Aldrich). The antibody was diluted in 'antibody diluent with background reducing components' (DAKO, Hamburg, Germany) at room temperature and incubated with the sections for $90 \mathrm{~min}$. After washing thrice in phosphate-buffered saline, peroxidase-coupled secondary antibody (Calbiochem, Darmstadt, Germany) was applied for $30 \mathrm{~min}$. Bound antibody was detected with 3-amino9-ethylcarbazole (Sigma Chemicals, Munich, Germany). All sections were then counterstained with hemalaun. For quantification, positive cells in parenchymal regions were counted in five different high-power fields of each sample.

\section{Caspase-3 Activity}

Measurement of liver caspase-3 activity was performed using the Caspase-3 Colorimetric Assay Kit (Axxora, Lörrach, Germany), which was performed according to the manufacturer's guidelines. In this assay, cleavage of the artificial substrate releases $p$-nitroaniline (pNA). The pNA light emission was measured at $405 \mathrm{~nm}$. Homogenates were prepared using the supplied cell lysis buffer. To exclude incorrect measurements due to substrate depletion, different amounts of liver homogenates were tested.

\section{PARP-1 Cleavage}

Expression and endogenous caspase- 3 cleavage of PARP-1 in liver samples was assessed by western blot analysis using an antibody that recognizes the large uncleaved protein $(115 \mathrm{kDa})$ and the small cleaving fragment $(25 \mathrm{kDa})$. This cleavage of PARP-1 is caused by caspase- 3 activity. For detection, samples were analyzed using $10 \%$ gels.

\section{DNA Isolation and Electrophoresis}

To detect fragmented or apoptotic DNA (small DNA fragments) in liver samples, DNA was isolated from liver samples using the Apoptotic DNA Ladder Extraction Kit (BioVision, Mountain View, USA) according to the manufacturer's a
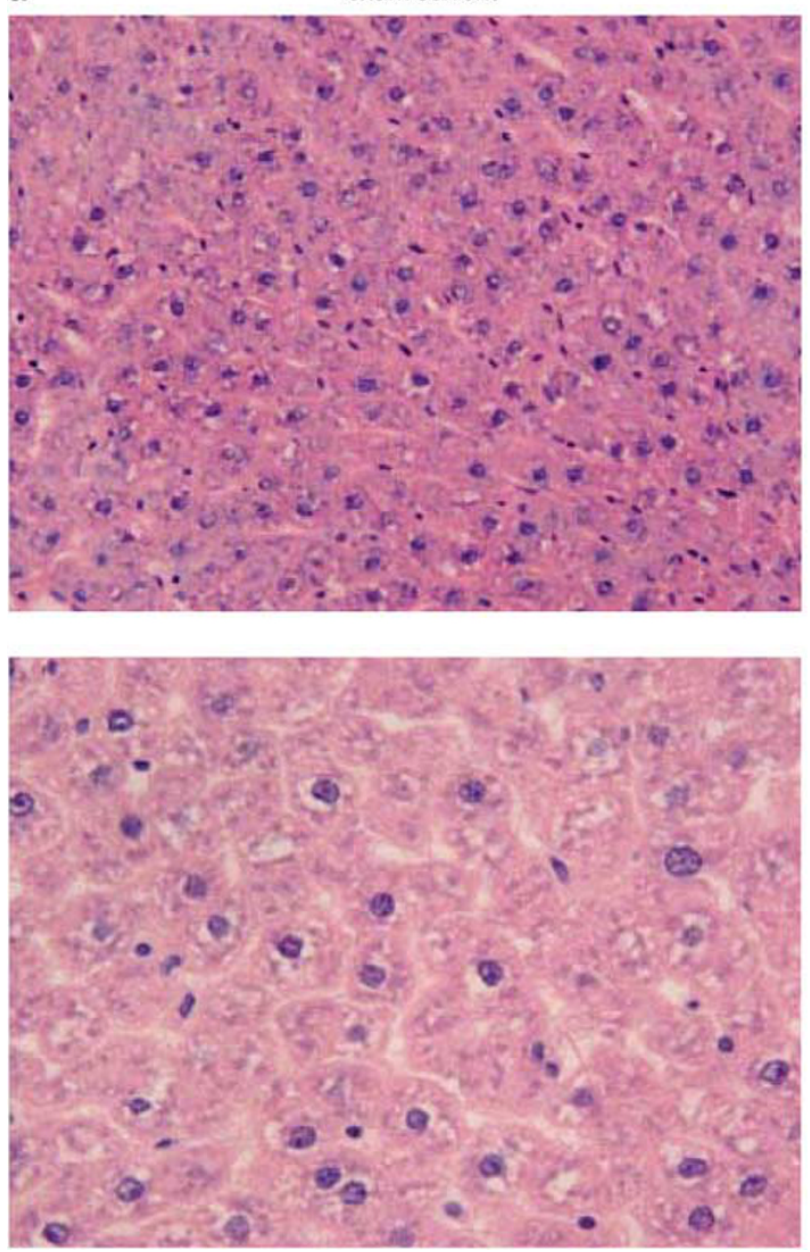

b
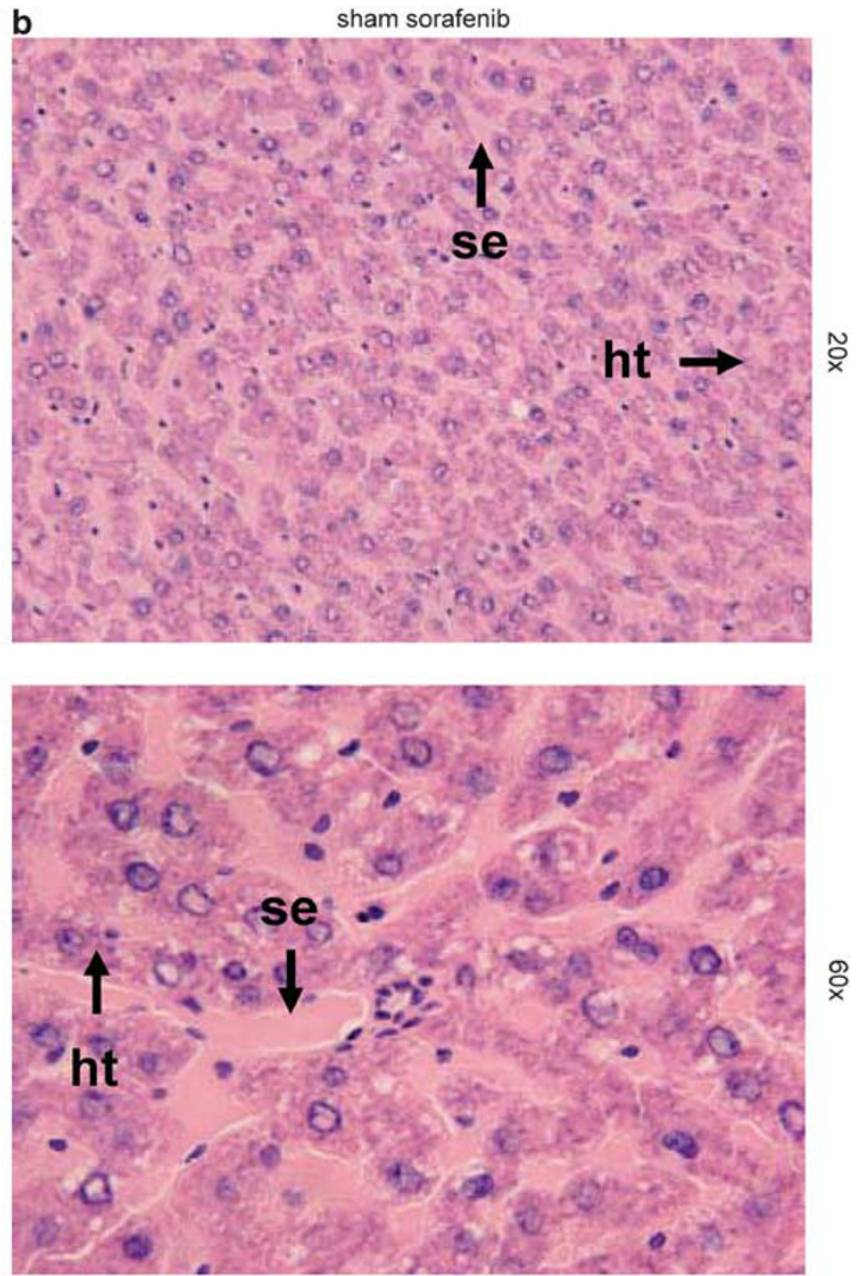

Figure 1 Effects of sorafenib on liver histology. (a) Liver tissue of solvent-treated sham-operated rats with normal hepatic architecture. (b) Liver tissue of sorafenib-treated sham-operated rats, with sinusoidal ectasia and congestion (se) and atrophy of hepatic trabeculae (ht). (c) Liver tissue of solvent-treated BDL rats with characteristic replacement of liver parenchyma by excessive bile duct formation (bd), vascular remodeling (see also Figure 4), and extracellular matrix deposition (see also Figure 5). (d) Liver tissue of sorafenib-treated BDL rats with bile duct formation (bd). (a-d) HE staining of liver sections. Shown are representative pictures from experiments with $n=5$ rats for each group with similar results. 
C
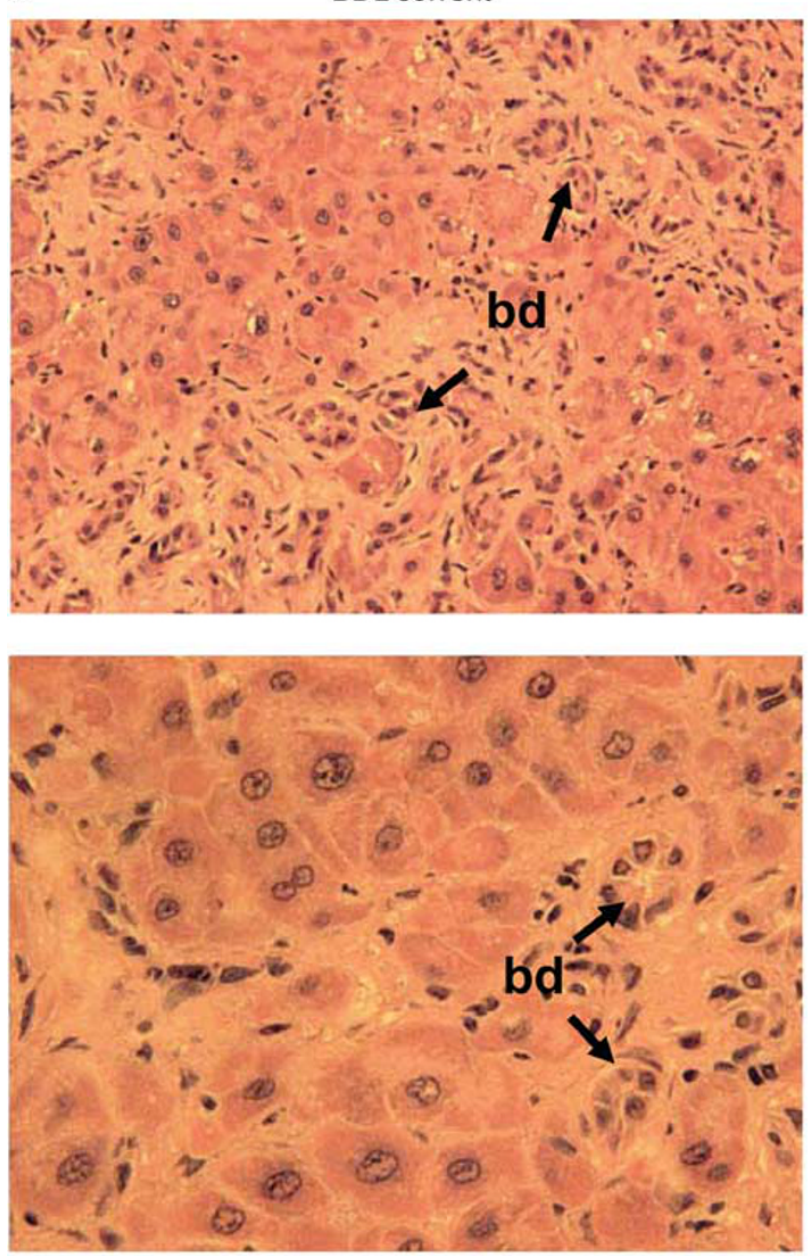

d
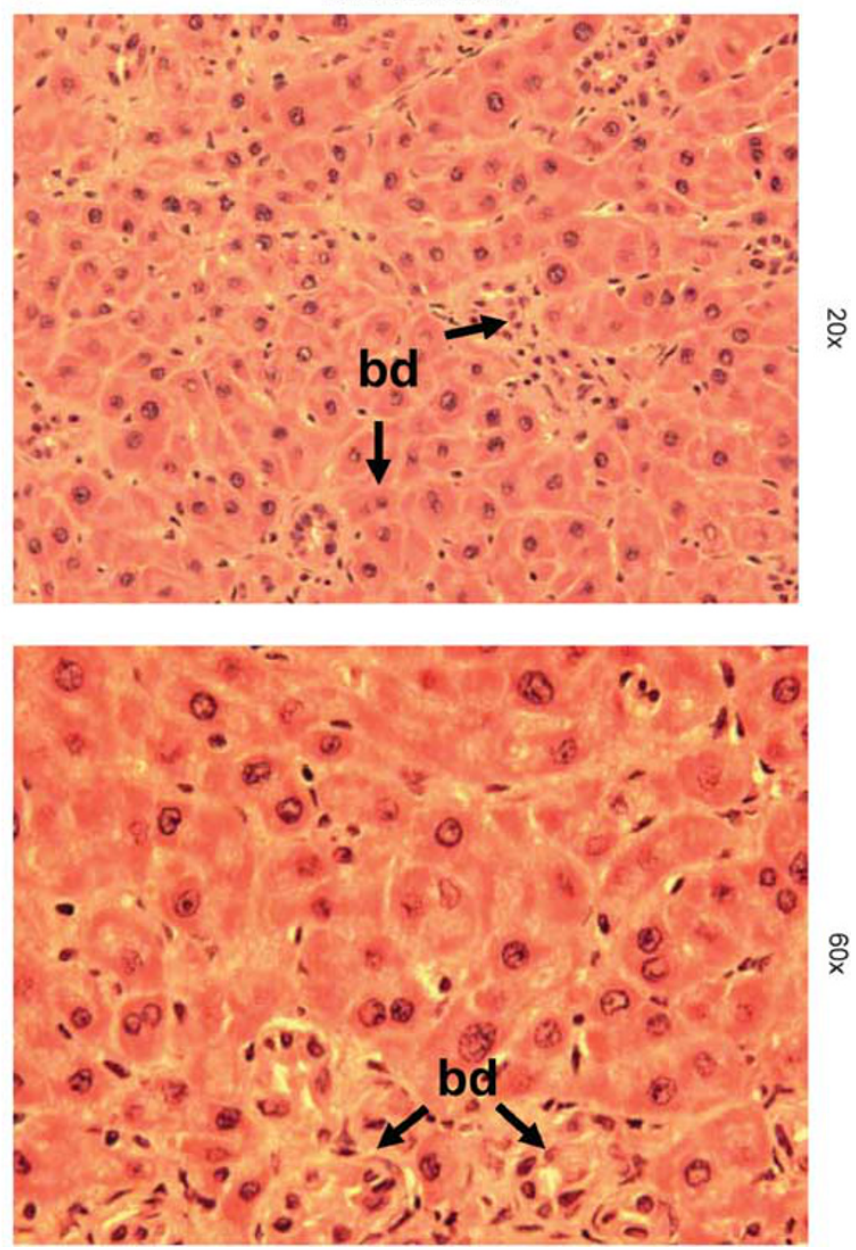

Figure 1 Continued.

guidelines. Samples were run in agarose gels, stained with ethidium bromide, and visualized by transillumination with UV light.

\section{Cell Isolation and Culture}

Hepatic stellate cells were isolated by in situ perfusion with a sequential collagenase type IV (Sigma, St Louis, USA) and pronase E (Merck, Darmstadt, Germany) solution from livers of normal rats as previously described. ${ }^{10}$ Characterization of the rat liver-derived myofibroblast-like cultures, established by culturing enriched HSC fractions on plastic, was performed by staining with anti- $\alpha \mathrm{SMA}$ antibody, as previously described. ${ }^{10}$ Experiments were performed between first and third passage (1:3 split ratio) using three independent cell lines. Cells were treated for 2 days either with sorafenib $(0.5$ or $1 \mu \mathrm{M})$ or with DMSO, which was used as solvent for in vitro experiments.

\section{Statistical Analysis}

Data are presented as means \pm standard error of the mean (s.e.m.) with the indicated number $(n)$ of experiments. $P$-values $<0.05$ were considered statistically significant.

\section{RESULTS Liver Histology}

HE staining revealed histological changes in livers of shamoperated (Figure 1a and b) and BDL rats (Figure 1c and d) induced by sorafenib, which differed between both groups (Figure 1). In livers of sham-operated rats, the treatment with sorafenib caused atrophy of hepatic trabeculae and induction of sinusoidal ectasia, resulting in sinusoidal overfilling and congestion (Figure 1a and b). Livers of solvent-treated BDL rats were characterized by partial replacement of liver parenchyma due to excessive bile duct formation, vascular remodeling, and deposition of ECM (Figure 1c). Bile duct formation was still observed in sorafenib-treated BDL rats (Figure 1d). Sinusoidal congestion or overfilling, as in shamoperated rats, was not observed in response to sorafenib treatment of BDL rats (Figure 1d).

\section{Biochemical Serum Parameters}

Analysis of biochemical serum parameters suggested the occurrence of sorafenib-induced liver damage in shamoperated and BDL rats (Figure 2). Liver damage, impaired 

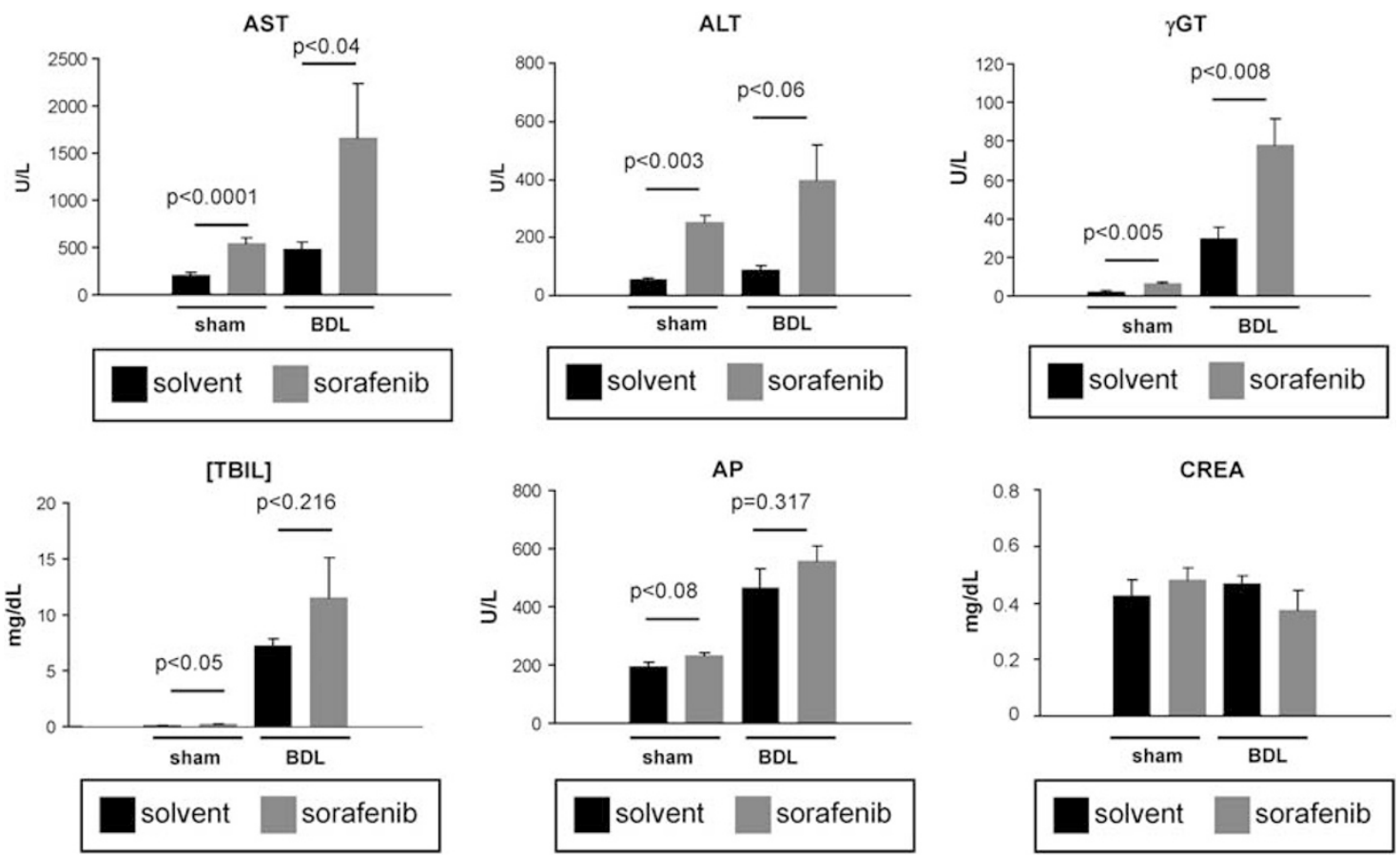

Figure 2 Biochemical analyses of serum parameters for liver and kidney function. Shown are data from solvent- and sorafenib-treated sham-operated and $\mathrm{BDL}$ rats. Data are means \pm s.e.m. ( $n=5$ for each group).
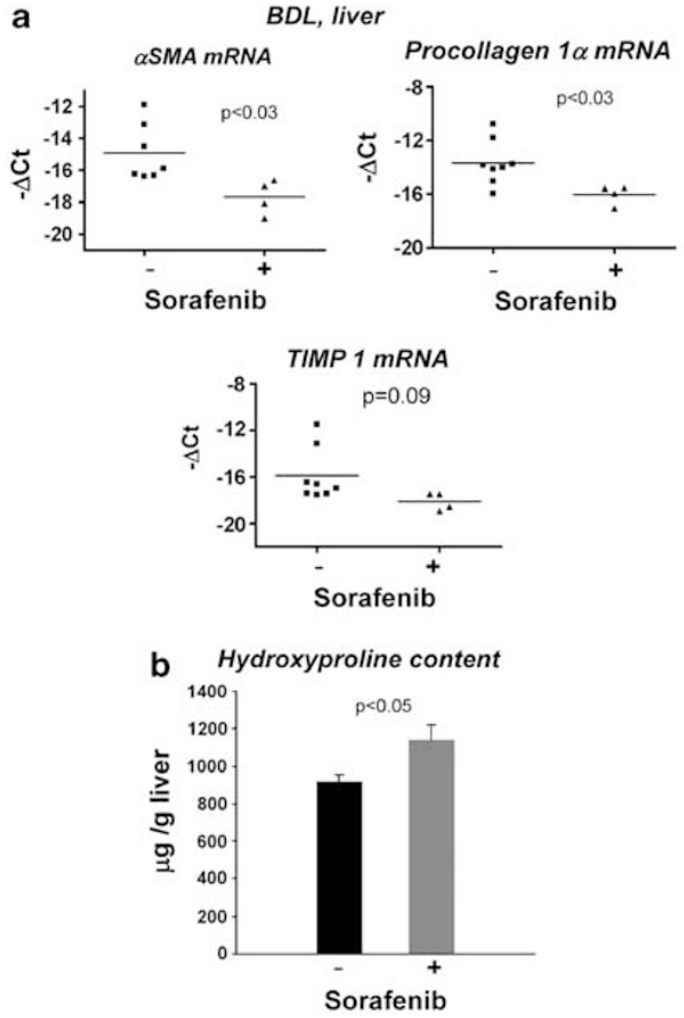

Figure 3 (a) Hepatic mRNA expression of $\alpha$ SMA, procollagen- $1 \alpha$, and TIMP 1 in solvent- and sorafenib-treated BDL rats. Shown are all results from RT-PCR experiments ( $n=4-8$ for each group). (b) Hepatic hydroxyproline content in solvent- and sorafenib-treated BDL rats. Data are means \pm s.e.m. from experiments with $n=5$ for each group. liver function, and cholestasis in solvent-treated BDL rats were reflected by elevations in liver enzymes and bilirubin (Figure 2). In sham-operated and in BDL rats, treatment with sorafenib caused hepatocellular damage, as shown by the sorafenib-induced increases in liver enzymes (Figure 2). This sorafenib-induced liver injury was enhanced in BDL rats (Figure 2). The sorafenib-induced increase in bilirubin may reflect an impairment of liver function (Figure 2). Changes in renal function were not noted, as reflected by the unaltered creatinine (Figure 2).

\section{Hepatic $\alpha$ SMA and Procollagen- $\alpha 1$ mRNA Expression}

Treatment of BDL rats with sorafenib reduced the hepatic expression of $\alpha$ SMA mRNA and procollagen- $\alpha 1$ mRNA (Figure 3a).

\section{Hepatic TIMP1 mRNA Expression}

Hepatic levels of TIMP1 mRNA remained unchanged by treatment of BDL rats with sorafenib (Figure 3a).

\section{Hepatic Hydroxyproline Content}

Treatment of BDL rats with sorafenib did not result in a reduction of the hepatic hydroxyproline content (Figure 3b).

\section{$\alpha$ SMA Staining}

Immunohistochemical staining of liver sections for $\alpha \mathrm{SMA}$ confirmed the typical expression of $\alpha$ SMA by perisinusoidal HSCs and by periportal and peribiliary myofibroblasts (Figure 4a). The increased number of these periportal, peribiliary and 
a

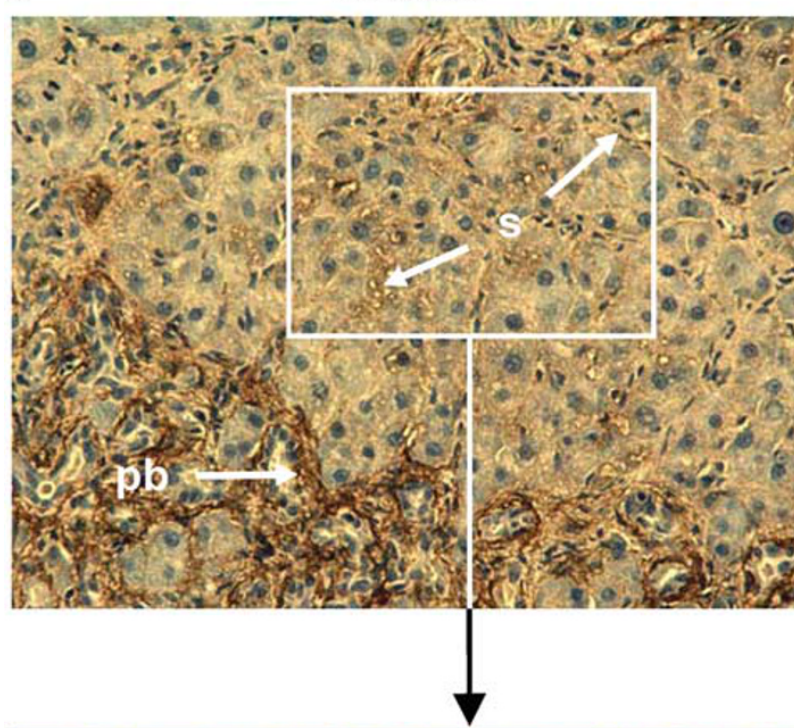

b

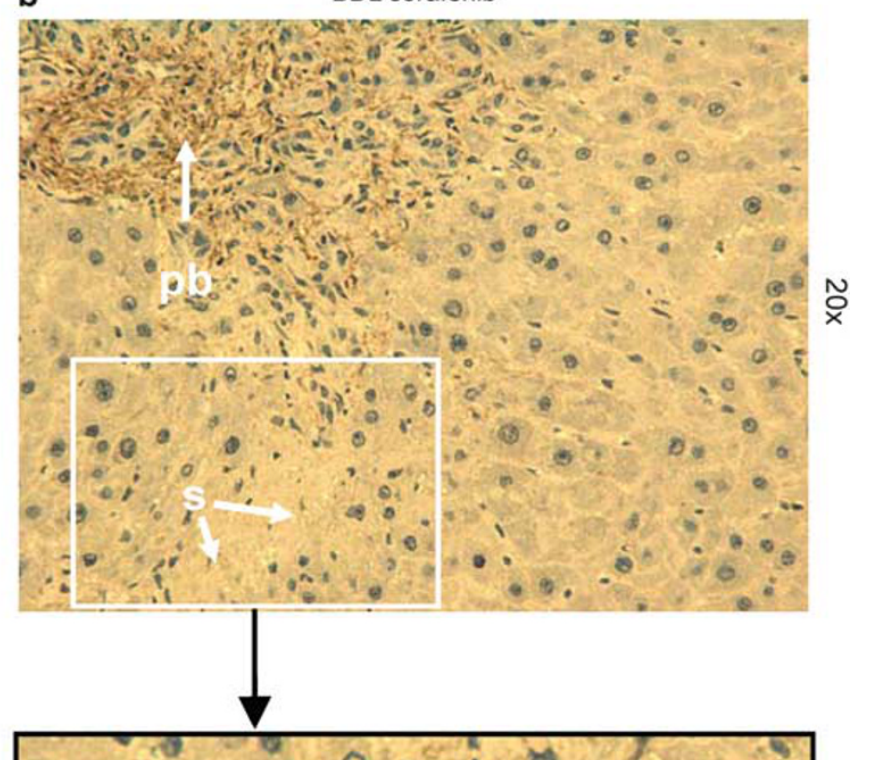

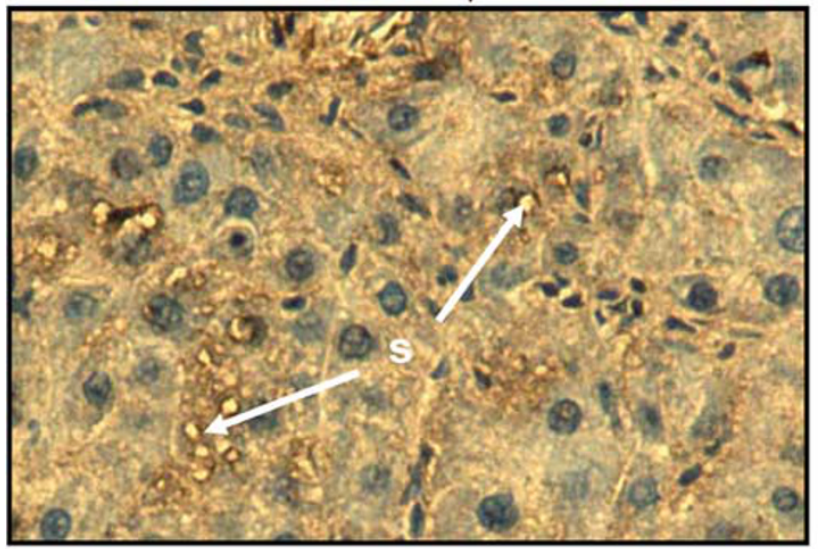
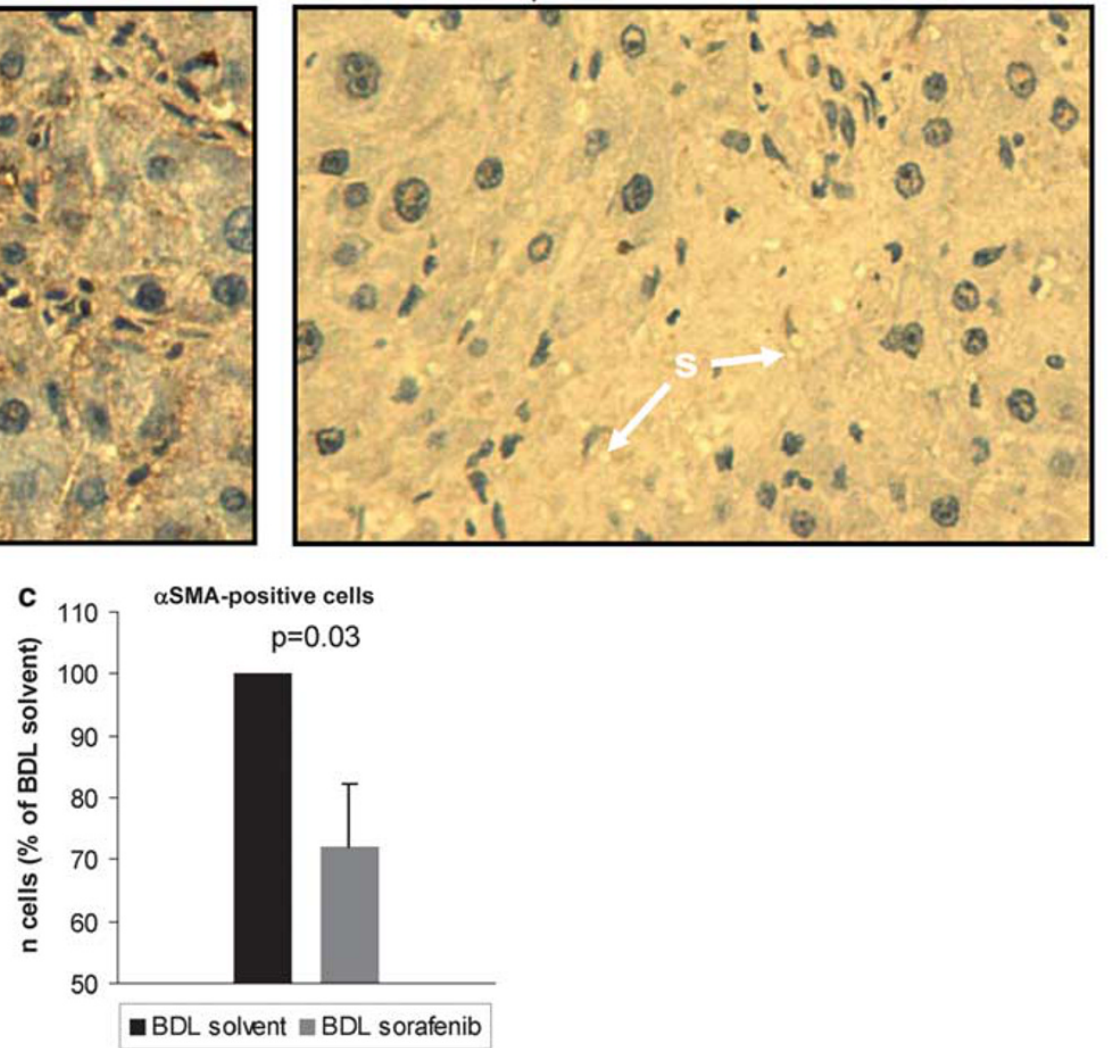

Figure 4 Comparison of $\alpha$ SMA expression in livers of solvent- and sorafenib-treated BDL rats. (a) Liver tissue of a solvent-treated BDL rat with strong perisinusoidal $(\mathrm{s})$ and peribiliary ( $\mathrm{pb}$ ) $\alpha \mathrm{SMA}$ staining (upper panel), and magnification of a region with strong perisinusoidal $\alpha \mathrm{SMA}$ staining (lower panel). (b) Liver tissue of a sorafenib-treated BDL rat without intrasinusoidal $\alpha$ SMA staining and weak peribiliary (pb) $\alpha \mathrm{SMA}$ staining (upper panel), and magnification of a region without perisinusoidal (s) $\alpha$ SMA staining (lower panel). (a and $\mathbf{b}$ ) Immunohistochemical $\alpha$ SMA staining of liver sections. Shown are representative pictures from experiments with $n=5$ rats per group with similar results. (c) Quantification of $\alpha$ SMA-positive cells in liver sections stained immunohistochemically for $\alpha$ SMA (data are means \pm s.e.m. from experiments with $n=5$ for each group).

perisinusoidal $\alpha$ SMA-positive intrahepatic cells in livers of $\mathrm{BDL}$ rats accompanied the formation of a vascular network in livers of BDL rats (Figure 4a). The treatment of BDL rats with sorafenib reduced the detectable $\alpha$ SMA-positive perisinusoidal vascular structures, but not the $\alpha \mathrm{SMA}$-positive structures in the periportal or peribiliary regions (Figure $4 \mathrm{~b}$ and $\mathrm{c}$ ). 
a

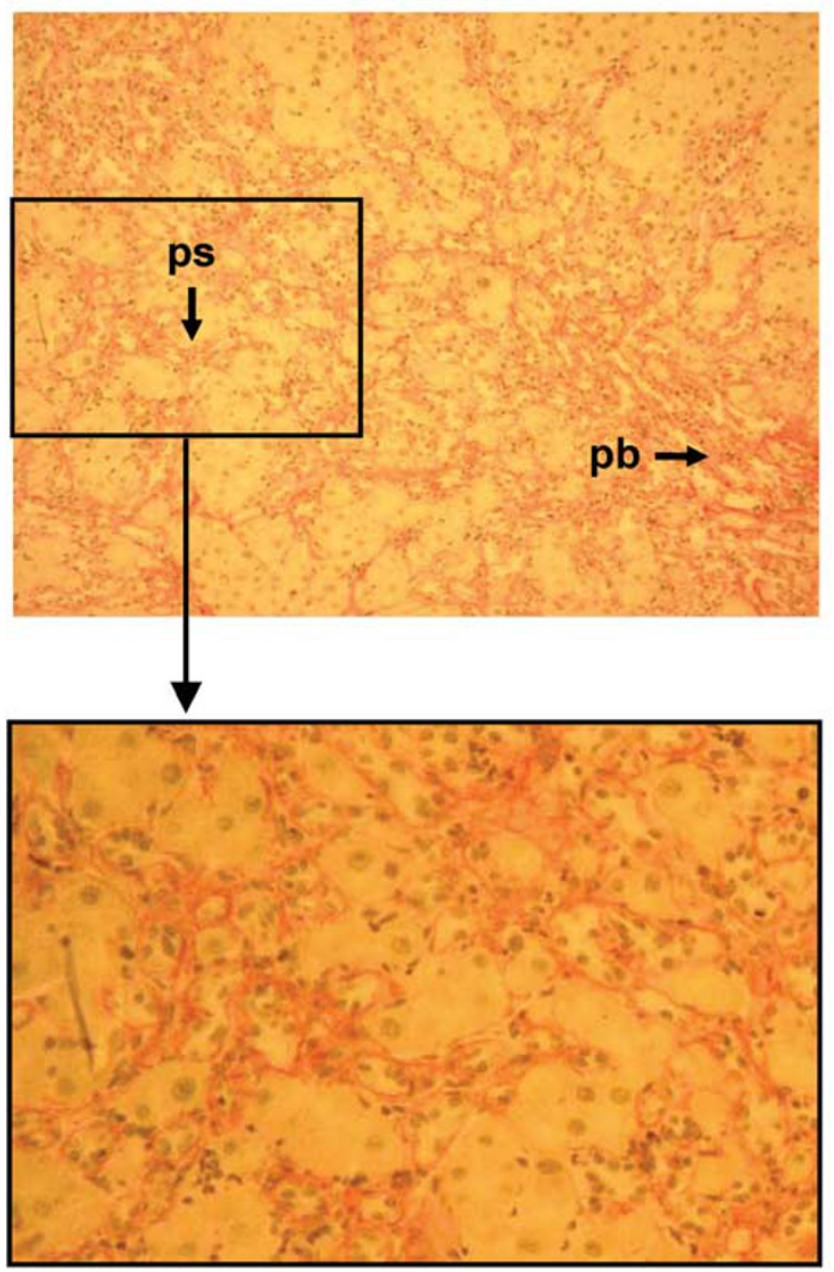

b

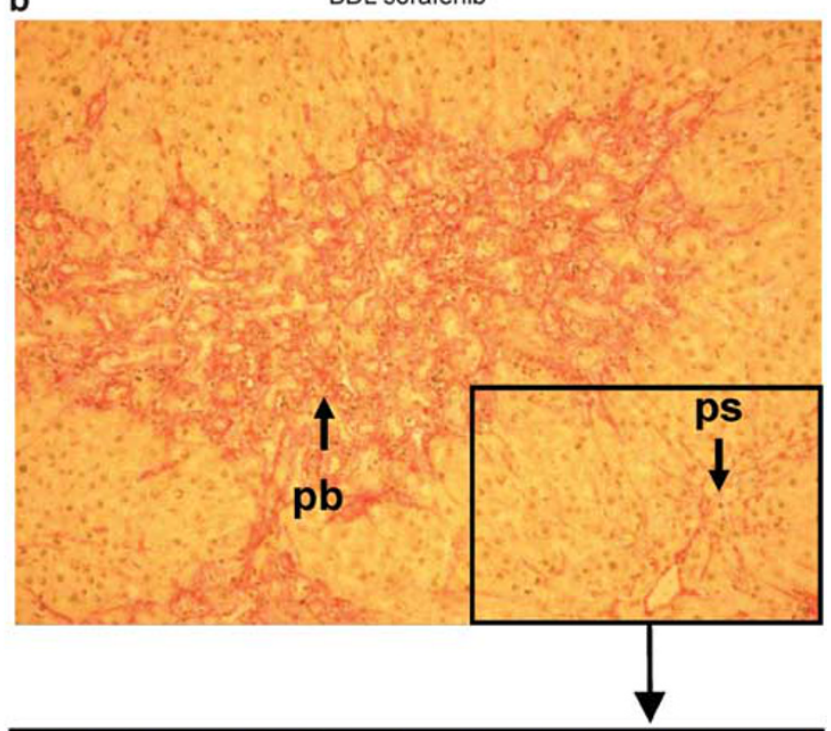

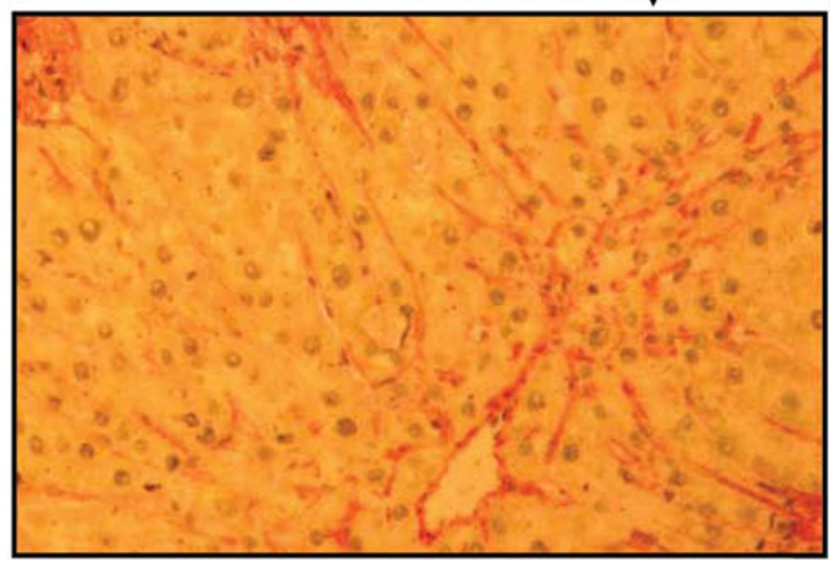

Figure 5 Hepatic deposition of extracellular matrix in solvent- and sorafenib-treated BDL rats. (a) Sirius red-stained liver section from a solvent-treated $\mathrm{BDL}$ rat, with perisinusoidal (ps) and peribiliary (pb) deposition of extracellular matrix (red) (upper panel), and magnification of a region with perisinusoidal Sirius red staining (lower panel). (b) Sirius red-stained liver section from a sorafenib-treated BDL rat, with discrete perisinusoidal (ps), and strong peribiliary (pb) deposition of extracellular matrix (red) (upper panel), and magnification of a region with discrete perisinusoidal Sirius red staining (lower panel). (a and b) Sirius red staining of liver sections. Shown are representative images from experiments with a total of $n=5$ solvent-treated BDL rats with similar results.

\section{Sirius Red Staining}

Sirius red staining of liver sections from solvent-treated BDL rats demonstrated typical perisinusoidal, periportal, and peribiliary fibrosis, resulting in the formation of fibrotic septae (Figure 5a).

As shown by Sirius red staining of liver sections from sorafenib-treated BDL rats, treatment of BDL rats with sorafenib caused resolution of perisinusoidal fibrosis, resulting in disappearance of fibrotic septae (Figure 5b). However, deposition of ECM was still observed in periportal and peribiliary areas (Figure 5b).

\section{Caspase-3 Activity}

As shown using a colorimetric enzyme assay, treatment of BDL rats with sorafenib was without significant effect on hepatic caspase-3 activity (Figure 6a).

\section{PARP-1 Cleavage}

As revealed by western blot analysis, cleavage of PARP-1 was increased in livers from BDL rats compared with shamoperated, as shown by the increased ratio of the small cleaving fragment and uncleaved PARP-1 (Figure 6b). No change in PARP-1 cleavage in livers of BDL or sham-operated rats was observed after treatment with sorafenib (Figure 6b). However, treatment with sorafenib resulted in upregulation of hepatic PARP-1 expression in BDL rats and downregulation of hepatic PARP-1 expression in sham-operated rats (Figure 6b).

\section{Small DNA Fragments}

Analysis of liver samples from sorafenib-treated BDL rats for small DNA fragments revealed small DNA fragments of considerably homogenous size $(\sim 150 \mathrm{bp})$ in samples from sorafenib-treated BDL rats (Figure 8). However, in samples 


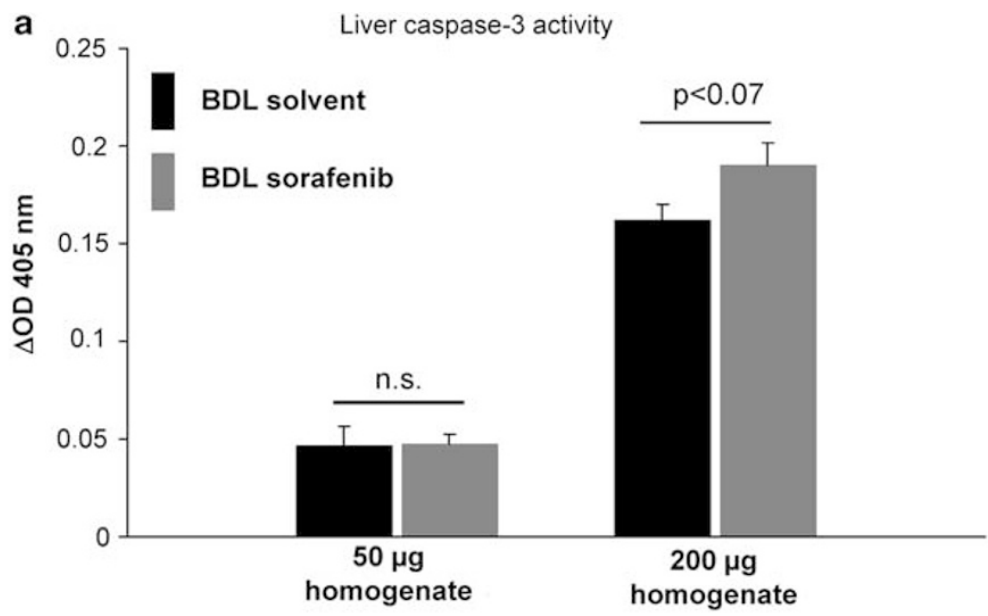

b
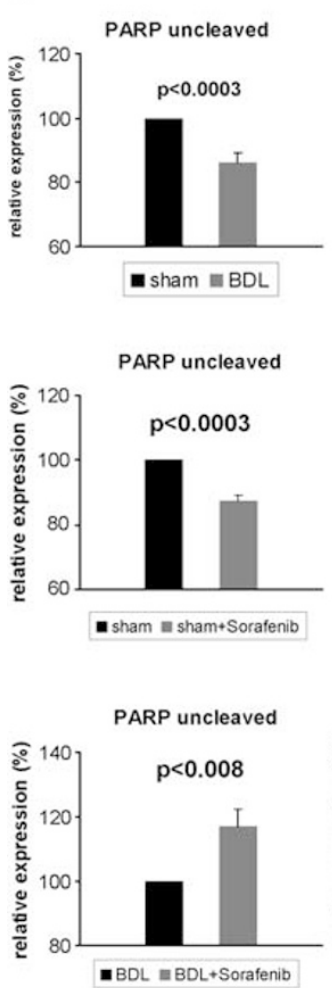

PARP small cleaving fragment
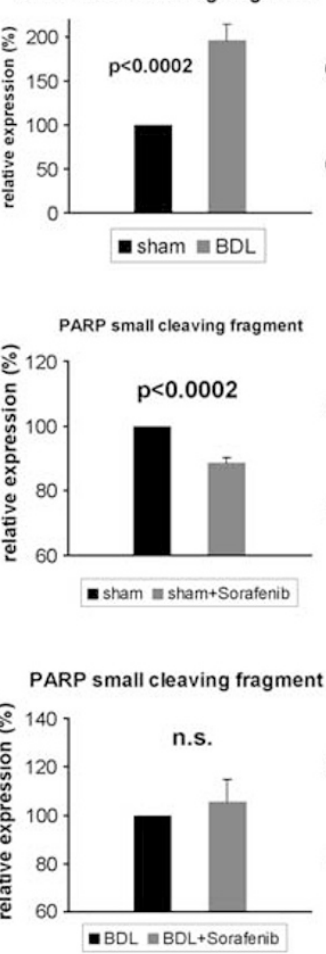

PARP-1 cleavage
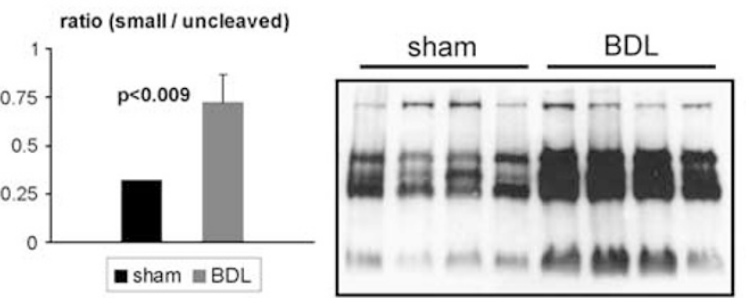

Uncleaved (115 kDa)

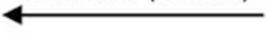

Small fragment (25 kDa)
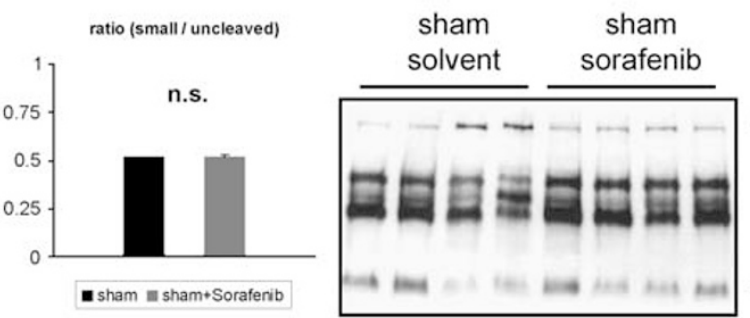

Uncleaved (115 kDa)
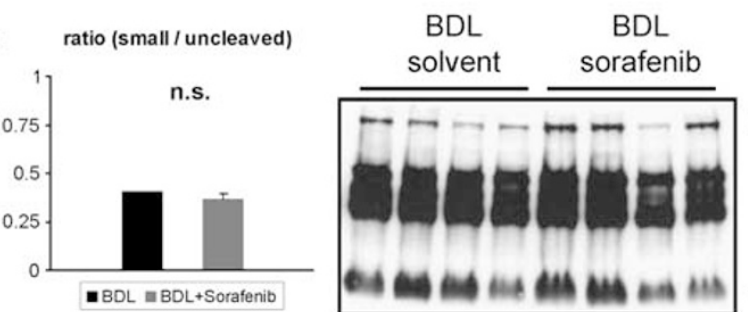

Uncleaved (115 kDa)

Small fragment (25 kDa)

$\longleftarrow$

Figure 6 Hepatic caspase-3 activity. (a) Caspase-3 enzyme activity on an artificial substrate in whole liver homogenates of solvent- and sorafenib-treated BDL rats. Data are means \pm s.e.m. from experiments with $n=6$ for each group. (b) Western blot analyses of the caspase- 3 substrate PARP-1 in livers of solvent- and sorafenib-treated rats. Shown are representative western blots and densitometric quantification of all experiments with $n=5$ solvent-treated and $n=5$ sorafenib-treated sham-operated rats, and $n=10$ solvent-treated and $n=10$ sorafenib-treated BDL rats. Quantification shows uncleaved PARP- 1 $(115 \mathrm{kDa})$, the small cleaving fragment $(25 \mathrm{kDa})$, and the ratio of both.

from untreated BDL rats, no such DNA fragments were detectable under the same conditions (Figure 7a).

\section{Hepatic Monocyte Chemoattractant Protein 1 mRNA Expression}

Hepatic levels of monocyte chemoattractant protein (MCP-1) mRNA remained unchanged by treatment of BDL rats with sorafenib (Figure 7b).

\section{Culture-activated Primary Rat HSCs}

Treatment of isolated, cultured HSCs from normal rats with sorafenib $(0.5$ or $1 \mu \mathrm{M})$ was without effect on morphology or number of HSCs (Figure 8a). The expression of $\alpha \mathrm{SMA}$ and procollagen- $1 \alpha$ mRNA in HSCs was assessed after 2 days of treatment with sorafenib or solvent. Both were dose-dependently reduced by sorafenib (Figure 8b). 


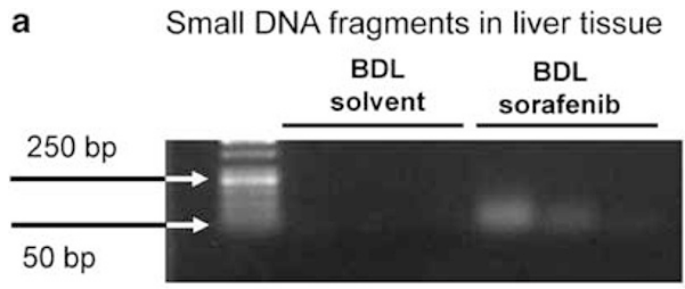

b

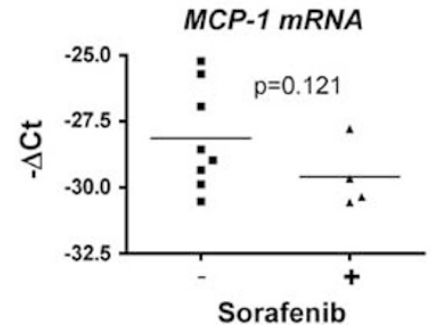

Figure 7 (a) Small DNA fragments in liver tissue of solvent- and sorafenibtreated BDL rats. Shown is a representative experiment with $n=3$ rats per group, from experiments with a total of $n=6$. (b) Hepatic mRNA expression of MCP-1 in solvent- and sorafenib-treated BDL rats. Shown are results from RT-PCR experiments ( $n=4-8$ for each group).

\section{DISCUSSION}

The multikinase inhibitor sorafenib was recently approved for the treatment of HCC in the European Union and the United States, and may be used in an increasing number of countries. ${ }^{711-14}$ Although treatment with sorafenib may be effective in patients with advanced HCC, ${ }^{7}$ little is known about its liver-specific effects in cirrhosis or on hepatic histology. ${ }^{11-13}$ Severe side effects may limit or even exclude therapy with sorafenib. ${ }^{12,13,15,16}$ On the other hand, tumorindependent benefits can not be excluded in sorafenib-treated patients. ${ }^{12,13}$ Recent studies in animal models of cirrhosis indicated that sorafenib ameliorates portal hypertension of cirrhotic rats via a decrease in intrahepatic vascular resistance. ${ }^{8,9}$ Here, we demonstrate that treatment with sorafenib modulates intrahepatic sinusoidal vascular remodeling in rats with established cirrhosis. This is explained by inhibition of activated HSCs, which consequently results in an antifibrotic effect. However, apart from this beneficial potential of sorafenib in cirrhosis, our results suggest that cirrhotic livers may be at a higher risk for sorafenib-induced liver damage than intact livers.

On the basis of our staining of liver sections with Sirius red, and for $\alpha \mathrm{SMA}$, we assume that sorafenib acted possibly more on HSCs than on other liver cells of BDL rats. The livers of our untreated BDL rats showed the characteristic distribution of $\alpha$ SMA-positive cells, which are activated HSCs close to the sinusoidal vascular bed, and vascular smooth muscle cells or pericytes in the periportal and peribiliary vessels. ${ }^{2,4,5}$ Density of $\alpha$ SMA-positive cells was highest in the peribiliary vascular plexus and along the sinusoids, indicating vascular remodeling and angiogenesis in these areas. ${ }^{2}$ Intrahepatic angiogenesis and remodeling of the intrahepatic vasculature in BDL rats is strongly connected to fibrosis, which includes extension of the sinusoidal vascular bed by activated and proliferating HSCs. ${ }^{2,4,5}$ Importantly, the treatment with sorafenib reduced perisinusoidal $\alpha$ SMA-positive cells, indicating a reduced number of activated HSCs. In contrast, the other vascular structures, that is, periportal and peribiliary vascular remodeling, remained unaffected by sorafenib. Hepatic MCP-1 expression was similar in solventand sorafenib-treated BDL rats. Therefore, we assume that the effect of sorafenib was caused by direct interventions in activated HSCs, but not by reducing of inflammation. MCP-1 may be produced by monocytes, macrophages, and biliary epithelial cells, ${ }^{17,18}$ and links hepatic inflammation to fibrosis and angiogenesis by HSC activation. ${ }^{6}$

Deposition of ECM by cells of the intrahepatic vascular bed characterizes cirrhosis and was presented by our BDL rats. Therefore, positive staining for Sirius red was highly abundant in the perisinusoidal regions of the parenchymal areas, in the peribiliary vascular plexus, and in the periportal regions. According to its effect on $\alpha \mathrm{SMA}$, sorafenib reduced perisinusoidal but not periportal or peribiliary fibrosis in our BDL rats. The reduced number of activated HSCs and the subsequent effect of sorafenib on fibrosis were further confirmed by analysis of whole liver homogenates and of isolated, cultured HSCs: sorafenib reduced hepatic mRNA of $\alpha \mathrm{SMA}$ and procollagen- $1 \alpha$ in vivo and in vitro in cultureactivated HSCs, indicating an inhibition of ECM synthesis. In contrast, hydroxyproline content and TIMP-1 expression remained unchanged by sorafenib, suggesting no effect on fibrinolysis. Our observation that sorafenib reduced the number of activated HSCs may be explained differently: (1) Sorafenib may induce apoptosis of HSCs, but not of other liver cells; (2) sorafenib may prevent the activation and/or the phenotype transformation of HSCs; or (3) sorafenib may alter the phenotype of activated HSCs.

Together, our investigations demonstrate a modulation of vascular remodeling in livers of BDL rats by sorafenib, which may be related to the antiangiogenic properties of sorafenib. As HSC activation and proliferation is highly responsible for intrahepatic angiogenesis and vascular remodeling in the cirrhotic liver, ${ }^{6}$ this effect of sorafenib is explained by an action on activated HSCs. Importantly, this is connected to the observed antifibrotic effect. An antifibrotic effect of sorafenib was recently suggested by another study, in which BDL rats were treated before fibrosis. ${ }^{9}$ In our current as well as our recent study, ${ }^{8}$ rats were treated with sorafenib in fully established cirrhosis. Finally, a reduced number of activated HSCs may not only explain the reduction in ECM production but also the reduced vasoconstrictor responsiveness of in situ-perfused livers, which was described recently. ${ }^{8}$ Together, the antifibrotic effect and the restored vasoconstrictor responsiveness lead to a decrease in intrahepatic vascular resistance, which consequently results in a reduction of portal pressure. ${ }^{8,9}$

Apart from the antifibrotic effects in BDL rats, the treatment of BDL and sham-operated rats with sorafenib under 
Isolated HSC

Sorafenib
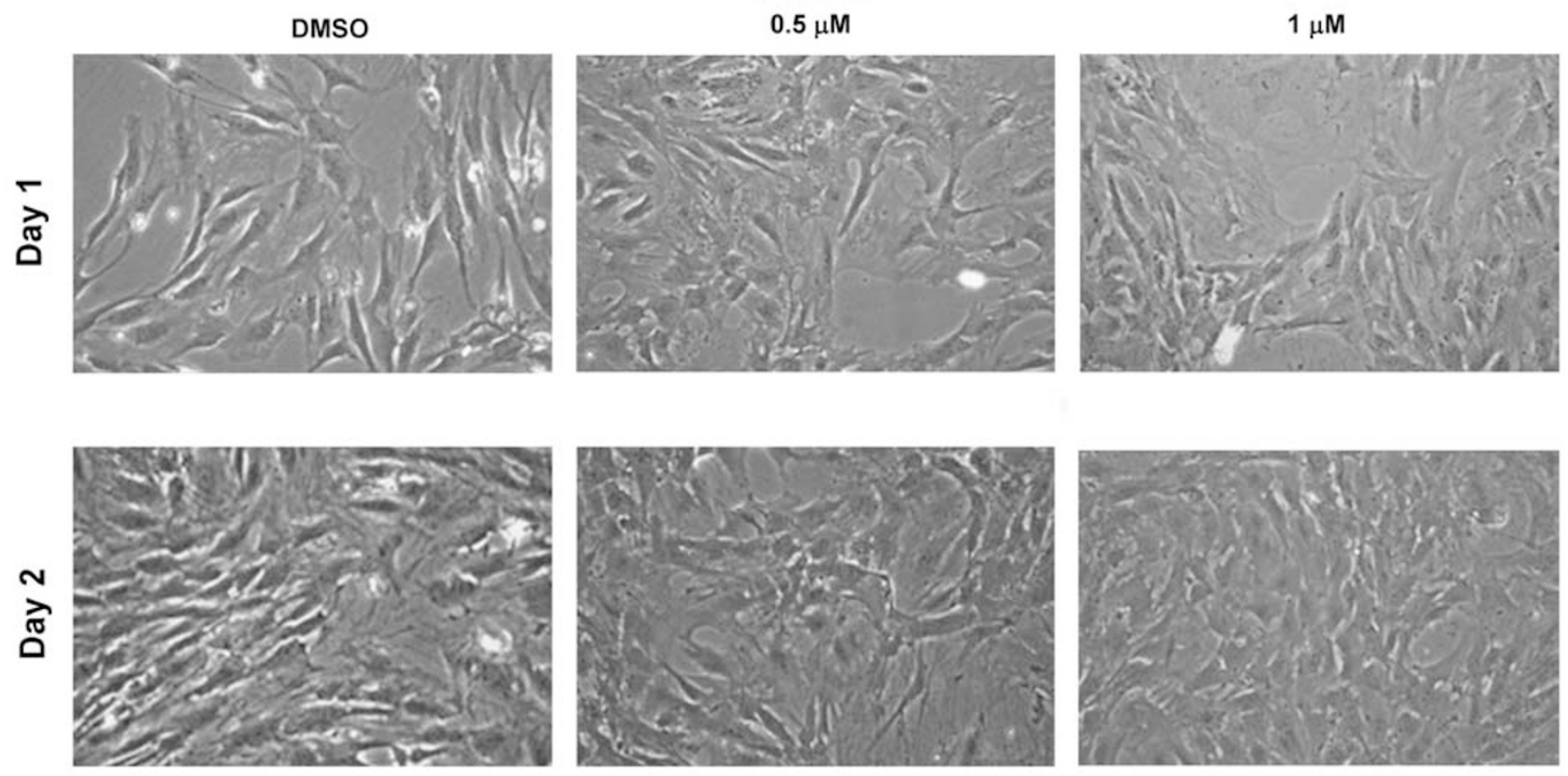

b 1.2

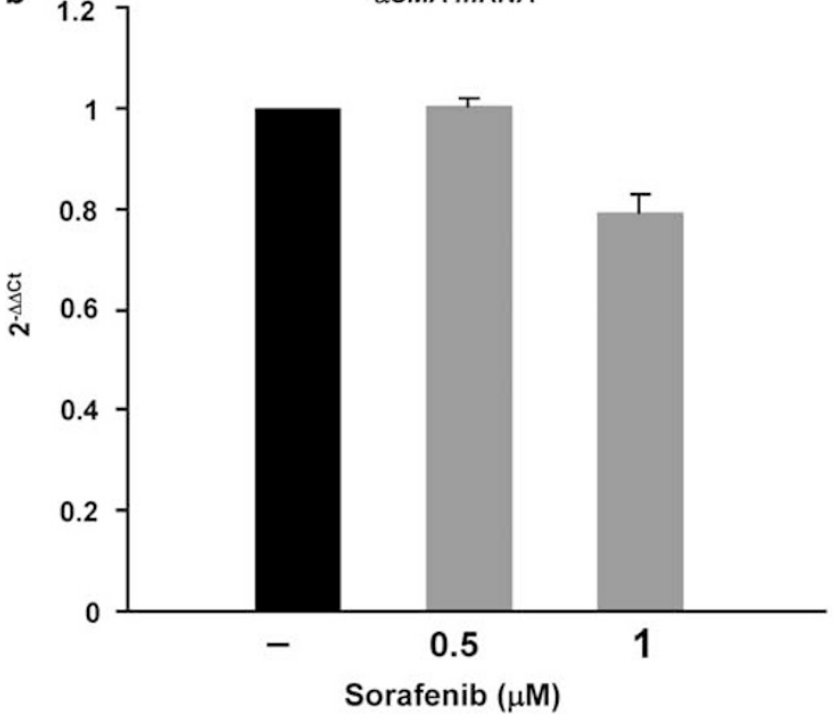

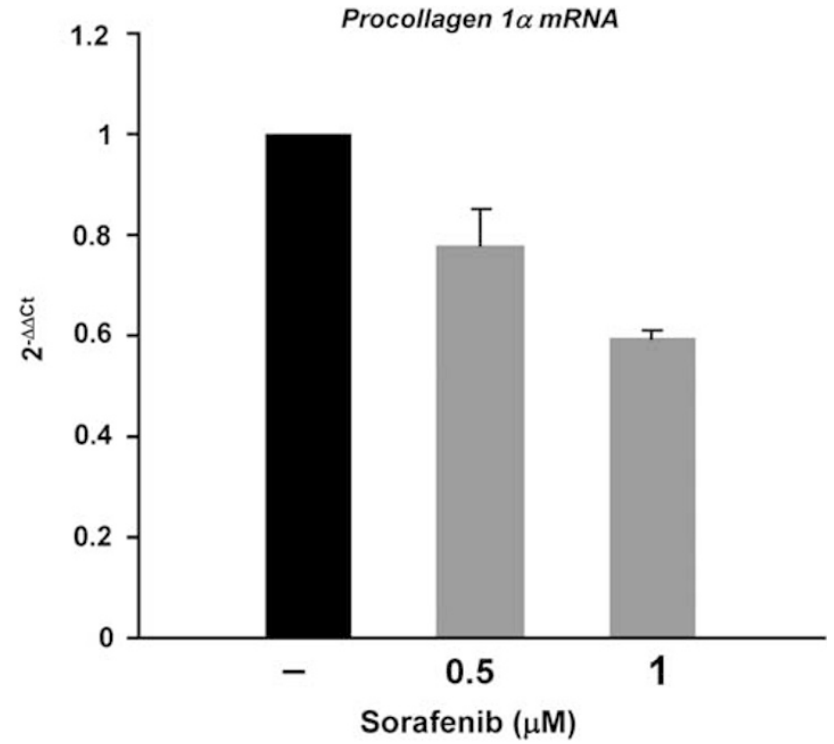

Figure 8 Effects of sorafenib in isolated, cultured rat HSCs. (a) Effect of sorafenib on morphology of isolated HSCs. Shown are representative images from experiments with HSCs isolated from three rats with similar results. (b) mRNA expression of $\alpha$ SMA and procollagen- $1 \alpha$ in DMSO- and sorafenib-treated $\mathrm{BDL}$ rats. Shown are data from experiments with HSCs isolated from three rats.

our conditions caused impairment of liver function, as indicated by biochemical analysis of blood serum parameters. This was more pronounced in BDL rats, which additionally showed typical impairment of liver function compared with their noncirrhotic controls. ${ }^{3}$ Our data suggest that any sorafenib-induced liver damage may be stronger in cirrhosis as in undamaged livers. After approval in the European Union and the United States, different trials identified sorafenibinduced alteration of liver function as a side effect. ${ }^{13,19}$ Thus, sorafenib-induced liver damage or hepatitis have been reported in two case reports. ${ }^{15,16}$ Different recent trials showed that treatment of HCC patients with sorafenib may require adaptations of the usual standard dose of $10 \mathrm{mg} / \mathrm{kg} / \mathrm{day}$ during therapy. ${ }^{7,12,13,14,19}$ Another study found rather high discontinuation rates in cirrhotic patients, which limited the applicability of sorafenib in these patients. ${ }^{12}$ Sorafenib was recommended for Child A patients, whereas the safety in patients with Child $\mathrm{B} / \mathrm{C}$ cirrhosis is still discussed. ${ }^{20}$ Adverse 
events include sorafenib-induced worsening of liver function, which is more frequent in patients with Child $\mathrm{B} / \mathrm{C}$ cirrhosis than in Child A patients. ${ }^{19}$ Our study underlines the possibility of sorafenib-induced liver damage. Although the dosage we used was rather high, liver toxicity of sorafenib in cirrhotic patients may occur at doses lower than that in our animal study. ${ }^{13,15,16,19}$ The dose in our study was based on antitumor studies performed in mice before the approval of sorafenib for the treatment of HCC. ${ }^{21}$

Apart from the changes of blood serum parameters, impairment of liver function by sorafenib was found in liver histology as well. Although conspicuous alterations of hepatocyte and sinusoidal morphology by sorafenib were the main findings in normal livers, these processes are covered by the cirrhosis-specific changes in the BDL model. Reduced size and mass of hepatocytes may explain the sorafenib-induced loss in liver function, and may be responsible for the sinusoidal ectasia and congestion in sham-operated rats. Sorafenib-induced damage of hepatocytes in BDL rats was reflected by changes in biochemical serum parameters. This finding is further supported by the appearance of small DNA fragments. Small DNA fragments were observed in livers from sorafenib-treated but not from solvent-treated BDL rats, and may result from sorafenib-induced hepatocyte damage. Together, sorafenib may act differentially in livers of BDL rats, although sorafenib-induced impairment of liver function is observed in both groups.

Owing to the beneficial effects of multikinase inhibitors on portal hypertension and fibrosis in animal models, ${ }^{8,9,22,23}$ sorafenib has been proposed as a new therapeutic option for treatment of portal hypertension. ${ }^{1}$ However, our present study together with recent clinical observations suggests that its translation to a new therapy and application in cirrhotic patients should be done with particular care. In patients with portal hypertension, the dosage of our study or standard doses may bear the risk of sorafenib-induced liver damage. However, dosage below the standard dose may effectively reduce portal pressure without injury. ${ }^{9}$

In conclusion, our findings point to HCC-independent effects, which may determine the prognosis of HCC patients under sorafenib therapy. Sorafenib may reduce the number of activated HSCs and thereby decrease intrahepatic vascular resistance by a dual mechanism: reduction of fibrosis and of vasoconstrictor responsiveness. However, our data suggest an increased risk for sorafenib-induced impairment of liver function in cirrhosis.

\section{ACKNOWLEDGEMENTS}

We thank G Hack, A Wollmann, S Bellinghausen, and C Conrad for excellent technical assistance, and S Dentler for critical reading of the paper. This study was supported by grants from the Deutsche Forschungsgemeinschaft (Grants HE 2402/5-3 and SFB TRR57, P18) and the Ernst und Berta GrimmkeStiftung (Grant 4/07).

\section{DISCLOSURE/CONFLICT OF INTEREST}

The authors declare no conflict of interest.
1. Shah V. Therapy for portal hypertension: what is our pipeline? Hepatology 2009;49:4-5.

2. Zhou Q, Hennenberg M, Trebicka J, et al. Intrahepatic upregulation of RhoA and Rho-kinase signalling contributes to increased hepatic vascular resistance in rats with secondary biliary cirrhosis. Gut 2006; 55:1296-1305

3. Trebicka J, Hennenberg M, Laleman W, et al. Atorvastatin lowers in portal pressure in cirrhotic rats by inhibition of RhoA/Rho-kinase and activation of endothelial nitric oxide synthase. Hepatology 2007;46: 242-253.

4. Reynaert $\mathrm{H}$, Thompson MG, Thomas $\mathrm{T}$, et al. Hepatic stellate cells: role in microcirculation and pathophysiology of portal hypertension. Gut 2002;50:571-581.

5. Friedman SL. Mechanisms of hepatic fibrogenesis. Gastroenterology 2008;134:1655-1669.

6. Lee JS, Semela D, Iredale J, et al. Sinusoidal remodeling and angiogenesis: a new function for the liver-specific pericyte? Hepatology 2007;45: 817-825.

7. Llovet JM, Ricci S, Mazzaferro V, et al. Sorafenib in advanced hepatocellular carcinoma. N Engl J Med 2008;359:378-390.

8. Hennenberg M, Trebicka J, Stark $C$, et al. Sorafenib targets dysregulated Rho kinase expression and portal hypertension in rats with secondary biliary cirrhosis. Br J Pharmacol 2009;157:258-270.

9. Mejias M, Garcia-Pras E, Tiani C, et al. Beneficial effects of sorafenib on splanchnic, intrahepatic and portocollateral circulations in portal hypertensive and cirrhotic rats. Hepatology 2008;49:1245-1256.

10. Trebicka J, Hennenberg M, Schulze Pröbsting A, et al. Role of beta3adrenoceptors for intrahepatic resistance and portal hypertension in liver cirrhosis. Hepatology 2009;50:1924-1935.

11. Zhu AX, Clark JW. Commentary: sorafenib use in patients with advanced hepatocellular carcinoma and underlying child-pugh B cirrhosis-evidence and controversy. Oncologist 2009;14:67-69.

12. Pinter $M$, Sieghart W, Graziadei I, et al. Sorafenib in unresectable hepatocellular carcinoma from mild to advanced stage liver cirrhosis. Oncologist 2009;14:70-76.

13. Yau T, Chan P, Ng KK, et al. Phase 2 open-label study of single-agent sorafenib in treating advanced hepatocellular carcinoma in a hepatitis B-endemic Asian population. Cancer 2009;115:428-436.

14. Cheng AL, Kang YK, Chen Z, et al. Efficacy and safety of sorafenib in patients in the Asia-Pacific region with advanced hepatocellular carcinoma: a phase III randomised, double-blind, placebo-controlled trial. Lancet Oncol 2009;10:25-34.

15. Schramm C, Schuch G, Lohse AW. Sorafenib-induced liver failure. Am J Gastroenterol 2008;103:2162-2163.

16. Llanos $L$, Bellot $P$, Zapater $P$, et al. Acute hepatitis in a patient with cirrhosis and hepatocellular carcinoma treated with sorafenib. Am J Gastroenterol 2009;104:257-258.

17. Marra F, DeFranco R, Grappone $C$, et al. Increased expression of monocyte chemotactic protein-1 during active hepatic fibrogenesis: correlation with monocyte infiltration. Am J Pathol 1998; 152:423-430.

18. Kruglov EA, Nathanson RA, Nguyen $T$, et al. Secretion of MCP-1/CCL2 by bile duct epithelia induces myofibroblastic transdifferentiation of portal fibroblasts. Am J Physiol Gastrointest Liver Physiol 2006;290: G765-G771.

19. Wörns MA, Weinmann A, Pfingst $K$, et al. Safety and efficacy of sorafenib in patients with advanced hepatocellular carcinoma in consideration of concomitant stage of liver cirrhosis. J Clin Gastroenterol 2009:43:489-495.

20. Peck-Radosavljevic M, Greten TF, Lammer J, et al. Consensus on the current use of sorafenib for the treatment of hepatocellular carcinoma. Eur J Gastroenterol Hepatol 2010;22:391-398.

21. Kim S, Yazici YD, Calzada G, et al. Sorafenib inhibits the angiogenesis and growth of orthotopic anaplastic thyroid carcinoma xenografts in nude mice. Mol Cancer Ther 2007;6:1785-1792.

22. Reiberger $T$, Angermayr $B$, Schwabl $R$, et al. Sorafenib attenuates the portal hypertensive syndrome in partial portal vein ligated rats. J Hepatol 2009;51:865-873.

23. Tugues S, Fernandez-Varo G, Munoz-Luque J, et al. Antiangiogenic treatment with sunitinib ameliorates inflammatory infiltrate, fibrosis, and portal pressure in cirrhotic rats. Hepatology 2007;46:1919-1926. 